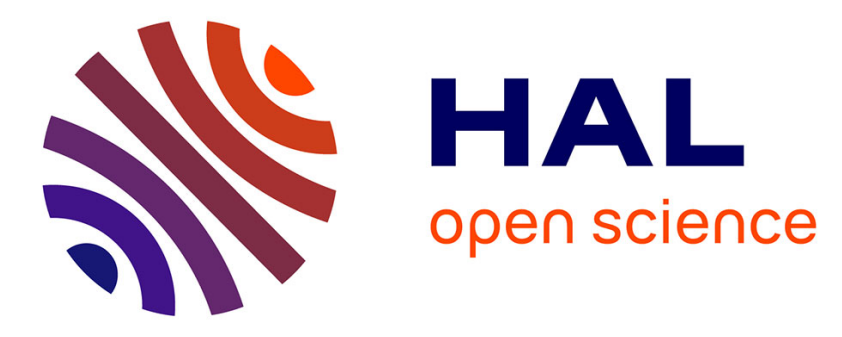

\title{
Swollen liquid-crystalline lamellar phase based on extended solid-like sheets
}

Jean-Christophe P. Gabriel, Franck Camerel, Bruno Jacques Lemaire, Hervé Desvaux, Patrick Davidson, Patrick Batail

\section{- To cite this version:}

Jean-Christophe P. Gabriel, Franck Camerel, Bruno Jacques Lemaire, Hervé Desvaux, Patrick Davidson, et al.. Swollen liquid-crystalline lamellar phase based on extended solid-like sheets. Nature, 2001, 413, pp.504-508. cea-03194654

\section{HAL Id: cea-03194654 https://hal-cea.archives-ouvertes.fr/cea-03194654}

Submitted on 9 Apr 2021

HAL is a multi-disciplinary open access archive for the deposit and dissemination of scientific research documents, whether they are published or not. The documents may come from teaching and research institutions in France or abroad, or from public or private research centers.
L'archive ouverte pluridisciplinaire HAL, est destinée au dépôt et à la diffusion de documents scientifiques de niveau recherche, publiés ou non, émanant des établissements d'enseignement et de recherche français ou étrangers, des laboratoires publics ou privés. 
might be expected in the vortex core of a high-temperature superconductor. If we interpret our relaxation rate in the same way as for quasiparticles outside the cores, following the discussion in the caption to Fig. 3 and equation (1), then both field and temperature dependence are opposite to what is required for the zero-bias anomaly. Consequently, instead of a peak in the density of states there is a mini-gap, $\sim \pm 5 \mathrm{meV}$, much sharper than the variation of the DOS outside the vortex core. Previous reports of vortex core states have been based on charge tunnelling in $\mathrm{YBa}_{2} \mathrm{Cu}_{3} \mathrm{O}_{7-\delta}$ (ref. 9) and $\mathrm{Bi}_{2} \mathrm{Sr}_{2} \mathrm{CaCu}_{2} \mathrm{O}_{8+\delta}$ (ref. 10). Near the Fermi energy $\left(\sim \pm 5.5 \mathrm{meV}\right.$ for $\left.\mathrm{YBa}_{2} \mathrm{Cu}_{3} \mathrm{O}_{7-\delta}\right)$, a mini-gap structure in the DOS was observed in the vortex core region. This result is inconsistent with theoretical predictions ${ }^{13}$. Furthermore, tunnelling is extremely sensitive to the surface and depends on an understanding of the tunnelling matrix elements ${ }^{14}$. In contrast, NMR probes bulk material. And yet both sets of experiments - the present work and refs 9, 10— can have a similar interpretation.

NMR is directly sensitive to the magnetic character of electronic excitations. So an alternative explanation of our results for the vortex core region might involve antiferromagnetism, as predicted by a theory ${ }^{2}$ that unifies superconductivity and antiferromagnetism. Then field-induced antiferromagnetic excitations could strongly enhance the NMR rate, consistent with our observations. In future work, spatially resolved NMR relaxation experiments could be extended to understand the effects of doping on vortex core states, and possibly identify a connection with the spin-pseudogap found in the normal state.

Received 25 April; accepted 13 August 2001.

1. Lake, B. et al. Spins in the vortices of a high-temperature superconductor. Science 291, 1759-1762 (2001).

2. Arovas, D. P., Berlinsky, A. J., Kallin, C. \& Zhang, S. C. Superconducting vortex with antiferromagnet core. Phys. Rev. Lett. 79, 2871-2874 (1997).

3. Takigawa, M., Ichioka, M. \& Machida, K. Theory of vortex excitation imaging via an NMR relaxation measurement. Phys. Rev. Lett. 83, 3057-3060 (1999).

4. Wortis, R., Berlinsky, A. J. \& Kallin, C. Spin-lattice relaxation in the mixed state of $\mathrm{YBa}_{2} \mathrm{Cu}_{3} \mathrm{O}_{7-\delta}$ and Doppler-shifted $d$-wave quasiparticles. Phys. Rev. B 61, 12342-12351 (2000).

5. Morr, D. K. \& Wortis, R. Theory of NMR as a local probe for the electronic structure in the mixed state of the high- $T_{c}$ cuprates. Phys. Rev. B 61, R882-R885 (2000).

6. Reyes, A. P. et al. Vortex melting in polycrystalline $\mathrm{YBa}_{2} \mathrm{Cu}_{3} \mathrm{O}_{7-\delta}$ from ${ }^{17} \mathrm{O}$ NMR. Phys. Rev. B 55, R14737-R14740 (1997).

7. Curro, N. J., Milling, C., Haase, J. \& Slichter, C. P. Local-field dependence of the ${ }^{17} \mathrm{O}$ spin-lattice relaxation and echo decay rates in the mixed state of $\mathrm{YBa}_{2} \mathrm{Cu}_{3} \mathrm{O}_{7-\delta}$. Phys. Rev. B 62, 3473-3479 (2000)

8. Hess, H. F., Robinson, R. B., Dynes, R. C., Valles, J. M. \& Waszczak, J. V. Scanning-tunnelingmicroscope observation of the Abrikosov flux lattice and the density of states near and inside a fluxoid. Phys. Rev. Lett. 62, 214-217 (1989).

9. Maggio-Aprile, I., Renner, Ch., Erb, A., Walker, E. \& Fischer, Ø. Direct vortex lattice imaging and tunneling spectroscopy of flux lines on $\mathrm{YBa}_{2} \mathrm{Cu}_{3} \mathrm{O}_{7-\delta}$. Phys. Rev. Lett. 75, $2754-2757$ (1995).

10. Pan, S. H. et al. STM studies of the electronic structure of vortex cores in $\mathrm{Bi}_{2} \mathrm{Sr}_{2} \mathrm{CaCu}_{2} \mathrm{O}_{8+\delta}$. Phys. Rev. Lett. 85, 1536-1539 (2000).

11. Volovik, G. E. Superconductivity with lines of gap nodes: density of states in the vortex. JETP Lett. 58, 469-473 (1993).

12. Lesueur, J., Greene, L. H., Feldmann, W. L. \& Inam, A. Zero bias anomalies in $\mathrm{YBa}_{2} \mathrm{Cu}_{3} \mathrm{O}_{7}$ tunneljunctions. Physica C 191, 325-332 (1992).

13. Franz, M. \& Tešanović, Z. Self-consistent electronic structure of a $d_{x^{2}-y^{2}}$ and a $d_{x^{2}-y^{2}}+i d_{x y}$ vortex. Phys. Rev. Lett. 80, 4763-4766 (1998)

14. Wu, C., Xiang, T. \& Su, Z. Absence of the zero bias peak in vortex tunneling spectra of hightemperature superconductors. Phys. Rev. B 62, 14427-14430 (2000).

15. Brandt, E. H. Precision Ginzburg-Landau solution of ideal vortex lattice for any induction and symmetry. Phys. Rev. Lett. 78, 2208-2211 (1997).

16. Mitrović, V. F., Sigmund, E. E. \& Halperin, W. P. Progressive saturation NMR relaxation. Phys. Rev. B 64, 024520-1-024520-7 (2001).

\section{Acknowledgements}

We thank J. A. Sauls, J. Moreno, R. Wortis and K. Machida for discussions. This work was supported by the Science and Technology Center for Superconductivity, the Materials Research Center at Northwestern Unviersity, and the National High Magnetic Field Laboratory supported by the National Science Foundation and the State of Florida. M.E. acknowledges support from the US Department of Energy, Office of Science.

\section{Swollen liquid-crystalline lamellar phase based on extended solid-like sheets}

\author{
Jean-Christophe P. Gabriel ${ }^{\star} \dagger$, Franck Camerel ${ }^{\star}$, Bruno J. Lemaire $\ddagger$, \\ Hervé Desvaux $₫$, Patrick Davidson $\ddagger$ \& Patrick Batail ${ }^{\star}$
}

* Sciences Moléculaires aux Interfaces, FRE 2068 CNRS, 2 rue de Houssinière, BP 32229, F-44322 Nantes Cedex 3, France

¥Laboratoire de Physique des Solides, UMR 8502 CNRS, Université Paris Sud, Bâtiment 510, Centre Universitaire, F-91405 Orsay Cedex, France

$\$$ Service de Chimie Moléculaire, URA 331 CEA/CNRS Saclay,

F-91191 Gif-sur-Yvette, France

Ordering particles at the nanometre length scale is a challenging and active research area in materials science. Several approaches have so far been developed, ranging from the manipulation of individual particles ${ }^{1,2}$ to the exploitation of self-assembly in colloids ${ }^{3}$. Nanometre-scale ordering is well known to appear spontaneously when anisotropic organic moieties form liquidcrystalline phases; this behaviour is also observed for anisotropic mineral nanoparticles ${ }^{4,5}$ resulting in the formation of nematic ${ }^{4-7}$, smectic $^{8}$ and hexagonal ${ }^{9,10}$ mesophases. Here we describe a lyotropic liquid-crystalline lamellar phase comprising an aqueous dispersion of planar solid-like sheets in which all the atoms involved in a layer are covalently bonded. The spacing of these phosphatoantimonate single layers can be increased 100-fold, resulting in one-dimensional structures whose periodicity can be tuned from 1.5 to 225 nanometres. These highly organized materials can be mechanically or magnetically aligned over large $\mathrm{pH}$ and temperature ranges, and this property can be used to measure residual dipolar couplings for the structure determination of biomolecules by liquid-state NMR. We also expect that our approach will result in the discovery of other classes of mineral lyotropic lamellar phases.

Lyotropic liquid-crystalline lamellar phases not only show longrange orientational order but also one-dimensional long-range positional order. They are classically formed (Fig. 1a-c) by selfassembly into layers of organic amphiphilic molecules, blockcopolymers or nanorods such as viruses or $\beta-\mathrm{FeOOH}$ colloids ${ }^{8,11}$. The inorganic layered materials that are used for intercalation purposes $^{12}$ display limited swelling (that is, their lamellar period can be increased) upon solvent addition. But these phases rarely show a lamellar period larger than a few nanometres, are hardly fluid and their positional order is lost very quickly upon further swelling. Therefore, such intercalation compounds cannot be considered as forming lamellar liquid-crystalline phases. With large swelling, concentrated aqueous gels of montmorillonite (smectite) clays may still display some long-range orientational ordering, as was recently demonstrated, but no positional long-range order was observed $^{13,14}$.

In our quest for a lyotropic liquid-crystalline lamellar phase comprised of purely inorganic solid-like sheets, we need to consider low-dimensional solid-state structures built from highly charged covalent single layers that can be swollen. These layers must be chemically stable upon swelling and have the smallest possible counter-ions. This is because electric charges are needed to produce long-range electrostatic repulsions and a small ion size is known to enhance solubility and swelling properties ${ }^{5}$. These criteria are met for the series of solid acids $\mathrm{H}_{n} \mathrm{M}_{n} \mathrm{Z}_{2} \mathrm{O}_{3 n+5}$ (with for example $\mathrm{M}=\mathrm{Sb}$, $\mathrm{Nb}, \mathrm{Ta} ; \mathrm{Z}=\mathrm{P}, \mathrm{As} ; n=1,3)$. Of these, we have chosen to study the 
layered phase, $\mathrm{H}_{3} \mathrm{Sb}_{3} \mathrm{P}_{2} \mathrm{O}_{14}$ (ref. 15; Fig. 1d). This phase is easily made in a three-step synthesis (the detailed, experimental procedure is given in the Supplementary Information), involving first the high-temperature solid-state synthesis of $\mathrm{K}_{3} \mathrm{Sb}_{3} \mathrm{P}_{2} \mathrm{O}_{14}$, then the exchange of potassium cations by protons in concentrated nitric

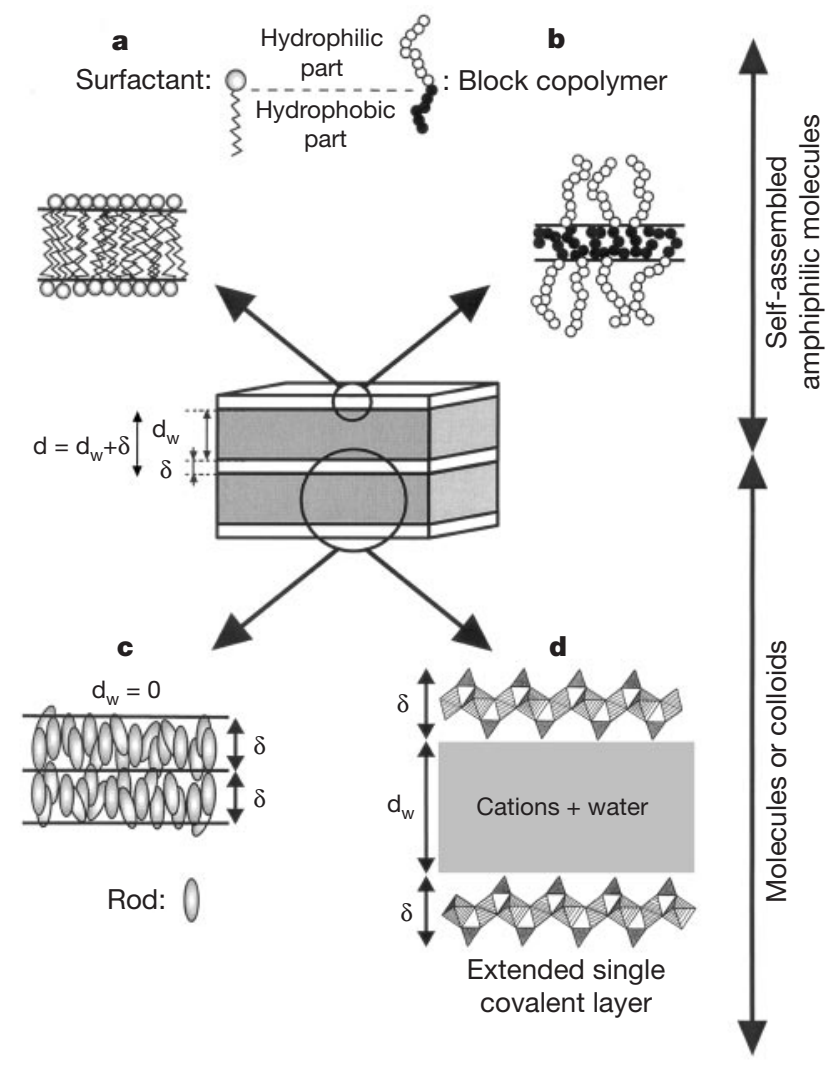

Figure 1 Possible architectures for a liquid crystalline lamellar phase. In a swollen lamellar phase (centre), the lamellae of thickness $\delta$ are regularly stacked with a period $d$ and separated by the solvent. The lamellae are usually made from the self-assembly of amphiphilic molecules, such as surfactants (a) or block copolymers $(\mathbf{b})$, or rod-shaped molecules or colloids (c). In contrast, the lamellae considered in this Letter are extended phosphatoantimonate covalent single layers made of at least 400 successive unit cells along any direction within the planes $(300 \mathrm{~nm})(\mathbf{d})$. The projection of the structure of $\mathrm{H}_{3} \mathrm{Sb}_{3} \mathrm{P}_{2} \mathrm{O}_{14}$ is also shown in $\mathbf{d}$ (dark tetrahedra represent $\mathrm{PO}_{4}$ groups and hatched octahedra represent $\mathrm{SbO}_{6}$ groups). acid $^{15}$, and finally, a thorough rinsing and dialysis of the obtained solid to remove remaining nitrate ions. This leads to homogeneous, transparent, colourless suspensions of $\mathrm{H}_{3} \mathrm{Sb}_{3} \mathrm{P}_{2} \mathrm{O}_{14}$ in water. Their viscoelastic properties depend on the overall mineral volume fraction, $\phi\left(\phi=M c / \rho\right.$; where $c$ is the concentration in $\mathrm{moll}^{-1}, M$ is the molecular mass in $\mathrm{g} \mathrm{mol}^{-1}$, and $\rho=2.4 \mathrm{~g} \mathrm{~cm}^{-3}$ is the density of $\mathrm{H}_{3} \mathrm{Sb}_{3} \mathrm{P}_{2} \mathrm{O}_{14}$ ), with dilute suspensions being very fluid.

Observation with the naked eye of a series of test-tubes containing suspensions of decreasing volume fraction between crossed polarizers shows that: (1) samples of high volume fraction $(\phi>1.78 \%$ ) form birefringent gels (Fig. $2 \mathrm{a}$ ); (2) for $0.75 \%<\phi$ $<1.78 \%$, fluid birefringent suspensions are observed (Fig. 2b); and (3) for $\phi<0.75 \%$ suspensions are biphasic with a well-defined interface between a denser birefringent fluid phase (bottom) and an isotropic, though flow birefringent, phase (top) (Fig. $2 \mathrm{c}$ and d). The existence of suspensions that are both very fluid and permanently birefringent unambiguously indicates the thermodynamic nature of the liquid-crystalline order. When examined in natural light the bottom phase shows interference colours ranging from blue to red as $\phi$ decreases (Fig. $2 \mathrm{~g}$ ). This already shows that there is some positional ordering at the length scale of fractions of a micrometre. This phase behaviour (Fig. 2) is that usually expected for the swelling of a lyotropic lamellar phase: once the maximum swelling is reached, water molecules can no longer be inserted into the interlamellar space and excess water is expelled, leading to phase separation. However, the flow birefringence of the top phase indicates that it contains some anisotropic particles in suspension. This can arise from an equilibrium between the lower and upper phases, which may be viewed as a colloidal 'evaporation/condensation' process, in analogy to a liquid/gas interface.

We established the phase diagram of $\mathrm{H}_{3} \mathrm{Sb}_{3} \mathrm{P}_{2} \mathrm{O}_{14}$ suspensions versus volume fraction and $\mathrm{NaCl}$ concentration (Fig. 3). Flocculation appears at large $\mathrm{NaCl}$ molarities $\left(>0.1 \mathrm{moll}^{-1}\right)$. The maximum lamellar period decreases with increasing salt concentration, inducing a shift of the lamellar phase boundary towards a higher volume fraction. Similarly, moving up a vertical line of the phase diagram in the biphasic domain, the volume of the lower birefringent phase decreases, showing that more water is expelled from the interlamellar space as the salt concentration increases. This is strong evidence that electrostatic repulsive interactions are needed to stabilize this lyotropic liquid-crystalline phase. Indeed, such an effect arises when additional salt ions screen the electrostatic forces, reducing their range.

The structure of these aqueous $\mathrm{H}_{3} \mathrm{Sb}_{3} \mathrm{P}_{2} \mathrm{O}_{14}$ suspensions has been further investigated by small-angle X-ray scattering (SAXS) on the

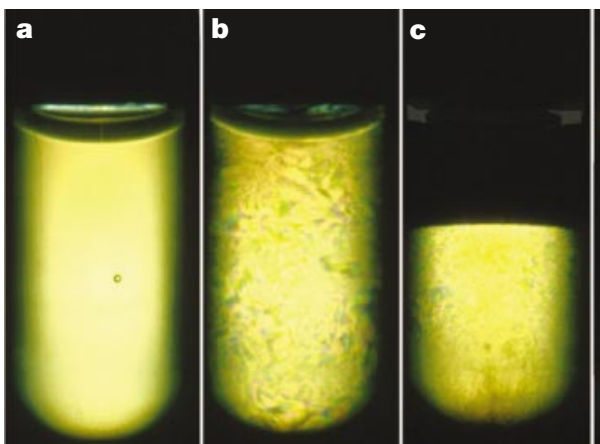

Figure 2 Naked-eye observation of samples. Test-tubes filled with aqueous suspensions of $\mathrm{H}_{3} \mathrm{Sb}_{3} \mathrm{P}_{2} \mathrm{O}_{14}$ single-layers, observed between crossed polarizers $(\mathbf{a}-\mathbf{e})$ (the isotropic phase in $\mathbf{c}$ and $\mathbf{d}$ appears dark). $\mathbf{a}$, in $2 \mathrm{ml}$ of birefringent gel phase (overall volume faction $\phi=1.98 \%$ ) the topological defects are so dense that the texture appears homogenous at the scale of this photograph. $\mathbf{b}, 2 \mathrm{ml}$ of birefringent fluid phase $(\phi=0.93 \%)$. c, $2 \mathrm{ml}$ of a biphasic sample $(\phi=0.65 \%)$. $\mathbf{d}, 2 \mathrm{ml}$ of a biphasic sample $(\phi=0.03 \%)$.
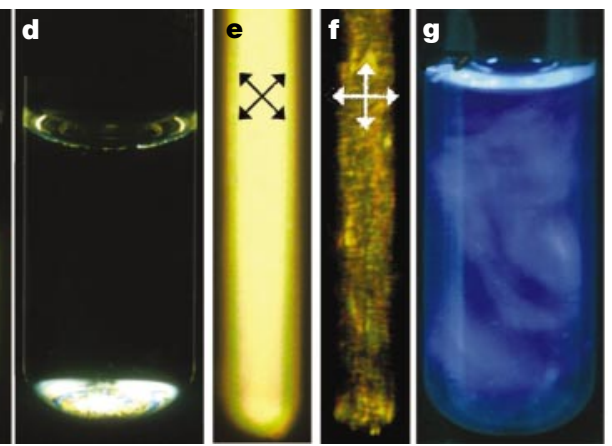

e and $\mathbf{f}$, Magnetically aligned sample observed in a 5-mm NMR tube that has been immersed for $10 \mathrm{~min}$ in a $18.7-\mathrm{T}$ field at $50^{\circ} \mathrm{C}$, in two different orientations compared to the polarizer//analyser system, represented by arrows $(\phi=0.75 \%)$. g. Sample iridescence $(\phi=0.75 \%$ ) observed in natural light is due to light scattering by the $\mathrm{H}_{3} \mathrm{Sb}_{3} \mathrm{P}_{2} \mathrm{O}_{14}$ layers stacked with a period of $225 \mathrm{~nm}$. 
ID2 high brilliance beamline at the European Synchrotron Radiation Facility $(E S R F)^{16}$. Experiments performed on very dilute isotropic suspensions showed an X-ray scattering intensity, $I$, that monotonously decreases with scattering vector modulus $q$ ( $q=(4 \pi \sin \theta) / \lambda$, where $2 \theta$ is the scattering angle) as $I \propto q^{-2}$ in all the range of concentrations studied $(0.033 \%<\phi<0.18 \%)$. This scattering law is typical of two-dimensional planar objects, and its $q$-range shows that sheets are of considerable lateral spatial extension (at least $300 \mathrm{~nm}$ ). These experiments prove that the layers remain flat in suspension and do not crumple or fold. At higher volume fractions, typical diffraction patterns of unoriented samples of birefringent suspensions (Fig. 4a) display many sharp peaks (up to 12) that can be indexed to the 001 reflections of a lamellar phase. The small width of these reflections proves that long-range positional ordering does take place, as expected for a lyotropic lamellar phase. The numerous higher-order reflections observed indicate that there is very little positional fluctuation ${ }^{17,18}$ and that the lamellar positional order is very high. Even at maximum swelling, quite a large number (up to 7) of lamellar reflections are still detected. This contrasts with more usual swollen lamellar phases comprised of liquid-like layers, and suggests that this lamellar phase comprised of solid-like sheets has very different elastic constants.

The evolution of the lamellar period $d$ of the birefringent phase upon dilution is shown in Fig. 4b. A first regime, $\phi>0.75 \%$, is observed in which $d \propto 1 / \phi$, as expected for the one-dimensional swelling of a pure lamellar phase. For $\phi \approx 0.75 \%$, a crossover to a second regime occurs as $d$ levels off and reaches a maximum of about $225 \mathrm{~nm}$. This regime corresponds to the expulsion of excess water in the biphasic domain of the phase diagram. The layer thickness $\delta \approx 1.05 \pm 0.05 \mathrm{~nm}$ can be extrapolated from the first regime, a value that compares very well with that derived from the crystallographic structure, $\delta \approx 1.10 \pm 0.01 \mathrm{~nm}$. This proves that the layers are perfectly exfoliated. Moreover, as expected, wide-angle Xray scattering experiments (on samples of volume fractions $\phi>1.78 \%$ ) showed the existence of fairly thin diffraction lines that arise from the long-range two-dimensional atomic positional order within the covalent layers (Fig. 4a, inset).

The major role of the electrostatic interactions was further

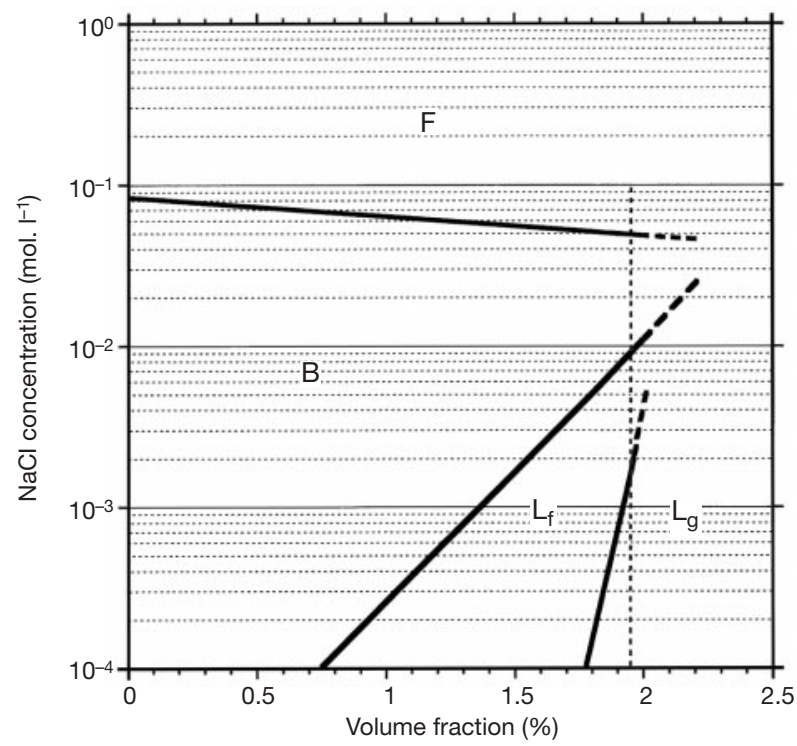

Figure 3 Phase diagram of $\mathrm{H}_{3} \mathrm{Sb}_{3} \mathrm{P}_{2} \mathrm{O}_{14}$ suspensions versus volume fraction and salt concentration. Upon decreasing the volume fraction, the suspensions first form a lamellar gel phase $\left(L_{g}\right)$, then a lamellar fluid phase $\left(L_{f}\right)$ and finally enter a biphasic regime $(B)$. The system flocculates $(F)$ at high salt molarity. assessed by SAXS experiments (Fig. 4c) in which the lamellar period was measured as a function of salt concentration, namely along the dashed line superimposed on the phase diagram (Fig. 3). $d$ strongly decreases at large ionic strength beyond $[\mathrm{NaCl}] \approx 10^{-2} \mathrm{moll}^{-1}$ when the electrostatic interactions are screened, as expected by the DLVO theory of colloidal stability ${ }^{19,20}$. Thus, at $\phi=1.9 \%, d$ falls from $40 \mathrm{~nm}$ in pure water down to $23 \mathrm{~nm}$ at $[\mathrm{NaCl}]=0.1 \mathrm{moll}^{-1}$.

The lamellar structure of these suspensions was confirmed by $\mathrm{X}$-ray scattering studies of single domains. Aligned samples could easily be produced by mechanical shearing with a Couette cell mounted on the ID2 beamline in both radial and tangential geometries (Fig. 5a), at shear rates, $\dot{\gamma}$, ranging from 30 to $1,000 \mathrm{~s}^{-1}$. At low volume fraction (near the biphasic domain), a strong alignment was induced even at very small shear rate (Fig. 5b). The three possible types of orientation ${ }^{21}$ were observed. However, we can conclude from the difference in scattering intensities that the intuitive $c$ orientation in which the layers orient parallel to the shearing surfaces, is very strongly dominant. The detection of some $a$ and $b$ orientations is very probably due to a few topological
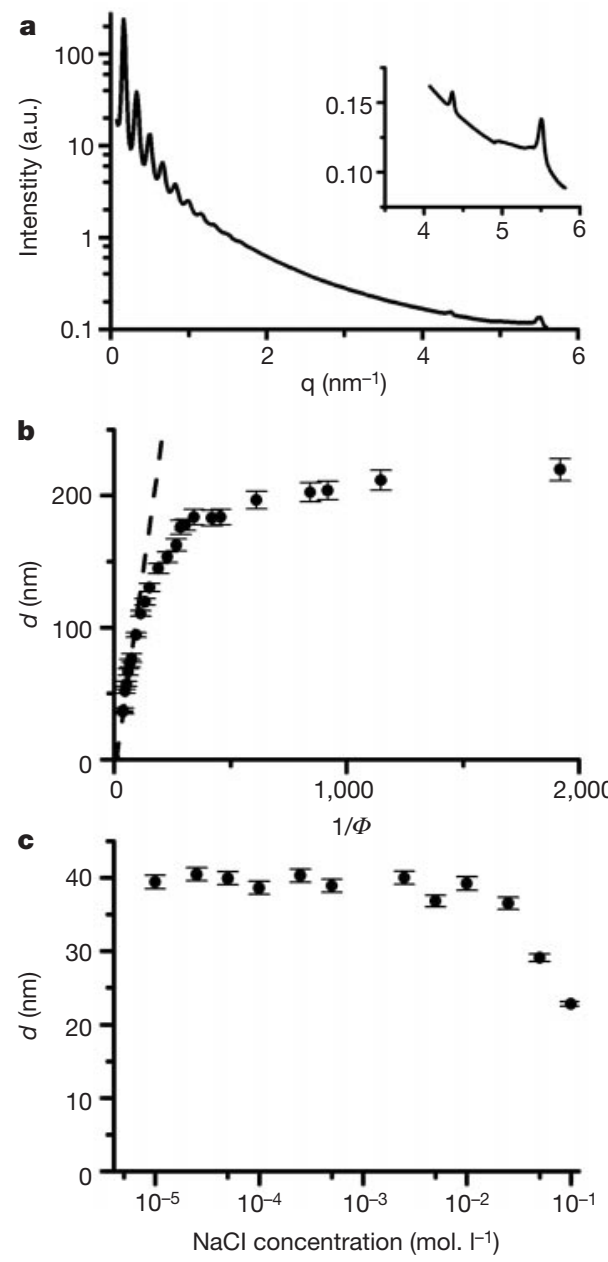

Figure 4 SAXS study of 'powder' samples. a, An example of scattered intensity versus scattering vector modulus $q$, showing 10 orders of reflection due to the lamellar period. Inset, the thin diffraction lines due to the two-dimensional positional order within the covalent layers $(\phi=2.0 \%)$. $\mathbf{b}$, Variation of the lamellar period, $d$, with inverse volume fraction (including two data points at very small $d$ extracted from ref. 16). The dashed straight line represents the one-dimensional swelling behaviour of the lamellar phase $d=\delta / \phi$ with $\delta=1.05 \mathrm{~nm}$. A crossover from this law to a plateau is observed when entering the biphasic regime. c, Variation of the lamellar period $d$ with salt molarity at constant volume fraction $(\phi=1.9 \%)$; along the vertical dashed line in Fig. 3. 
defects. No relaxation was observed when the shearing was stopped, which proves that the aligned domain is stable (Fig. 5b). Similarly, samples aligned by sucking them into capillary tubes still showed high orientation weeks after their preparation. Comparable results were also obtained with samples of larger volume fractions. However, the degree of alignment of the sheared samples decreased as their concentration increased, probably because of their larger viscoelasticity, which hinders alignment.

Single domains of aqueous suspensions of $\mathrm{H}_{3} \mathrm{Sb}_{3} \mathrm{P}_{2} \mathrm{O}_{14}$ could even be grown in magnetic fields classically used for NMR. Indeed, a SAXS study of these aligned samples showed mosaic spreads as low as $20^{\circ}$ (full-width at half-maximum, FWHM) (Fig. 5c) and proved that the layers align their normals parallel to the magnetic field. Also, ${ }^{2} \mathrm{H}$ NMR spectra obtained from suspensions of $\mathrm{H}_{3} \mathrm{Sb}_{3} \mathrm{P}_{2} \mathrm{O}_{14}$ in deuterated water, $\mathrm{D}_{2} \mathrm{O}$, exhibit a well-resolved residual quadrupolar splitting of the water signal. This property of magnetic alignment, observed in the whole temperature range useful for biomolecules $\left(4-50{ }^{\circ} \mathrm{C}\right)$, could easily be extended at any $\mathrm{pH}$ in the range $2.5-9.5$ by using a dilute base $\left(c<0.3 \mathrm{moll}^{-1}\right)$ with a bulky counter-ion (to avoid flocculation) such as tetramethylammonium hydroxide or tetra( $n$-butyl)ammonium hydroxide (Fig. 2e, f). This medium induces a partial alignment of the dissolved biomolecules, as illustrated in Fig. 6 on an oligosaccharide, leading to residual dipolar couplings, which provide long-range structural restraints that help to determine the structure of the biomolecule by solutionstate $\mathrm{NMR}^{22}$. The alignment tensor is significantly different from that induced by vanadium pentoxide suspensions $s^{23}$, so the simultaneous use of these two mineral liquid crystals is very useful for structure determination $^{24}$ (see Supplementary Information). By taking advantage of the slow kinetics of the phase separation (a few days), we could even prepare single domains, as observed optically and by NMR, at volume fractions inside the biphasic domain. This procedure could be used to tune the degree of alignment of biomolecules over an even larger range of concentrations. Finally, the possibility of lyophilizing the whole system over a wide range of $\mathrm{pH}$, and the fact that tetramethylammonium hydroxide is commercially available in perdeuterated form, provides a ${ }^{1} \mathrm{H} /{ }^{13} \mathrm{C} /{ }^{15} \mathrm{~N}$-free liquid crystal that seems particularly promising for the structural determination by liquid-state NMR of non-labelled biomolecules.

From a more fundamental perspective, the symmetry properties of this mineral lyotropic lamellar mesophase are very unusual: it has orientational order and exhibits a periodic one-dimensional stacking of covalent solid-like layers. The strong covalent bonds that
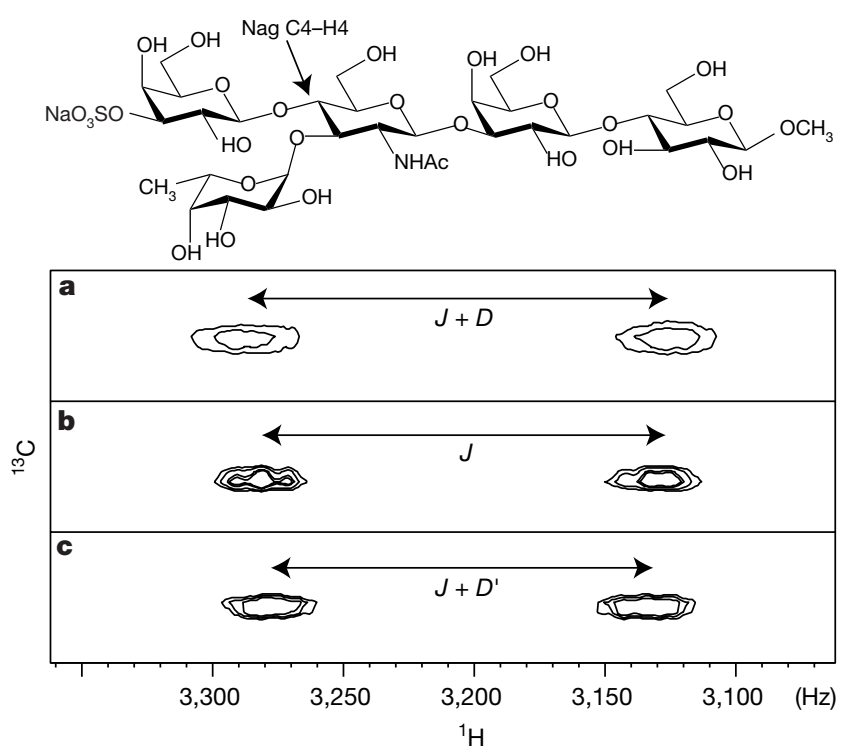

Figure 6 Superposition of three non-decoupled ${ }^{13} \mathrm{C}-{ }^{1} \mathrm{H}$ NMR sub-spectra ${ }^{29}$ of the glucosamine ( $\mathrm{Nag}$ ) $\mathrm{C} 4-\mathrm{H} 4$ region of the pentasaccharide containing the Lewis ${ }^{\mathrm{X}}$ motif acquired in a suspension of $\mathrm{V}_{2} \mathrm{O}_{5}(\mathbf{a})$, in isotropic $\mathrm{D}_{2} \mathrm{O}$ solution (b) and in a suspension of $\mathrm{H}_{3} \mathrm{Sb}_{3} \mathrm{P}_{2} \mathrm{O}_{4}$ in $\mathrm{D}_{2} \mathrm{O}, \phi=0.75 \%$ (c). In a and $\mathbf{c}$, the residual dipolar coupling $D=15.7 \mathrm{~Hz}$ and $D^{\prime}=-5.2 \mathrm{~Hz}$ superimposes to the scalar $J$ coupling, confirming the induced alignment of the biomolecule. The different $D$ values obtained using these two media illustrate the different induced alignments (see Supplementary Information).

make up the layers of this mineral lamellar phase are reminiscent of the $\mathrm{L}_{\beta}$ phase of amphiphilic molecules ${ }^{25}$. However, one important difference is that the layers of the $\mathrm{L}_{\beta}$ phase form through the crystallization of self-assembled amphiphilic molecules and melt on heating. In contrast, the mineral lamellar phase, even when swollen, is not sensitive to temperature (up to $70^{\circ} \mathrm{C}$ where chemical degradation starts taking place within days), owing to the strength of the covalent bonds. This lamellar phase of extended single layers is also an interesting experimental system for testing theoretical predictions describing the properties of periodic stacks of tethered membranes. Indeed, the statistical physics of membranes made up from strong covalent bonds are expected to be very different from those of liquid-like
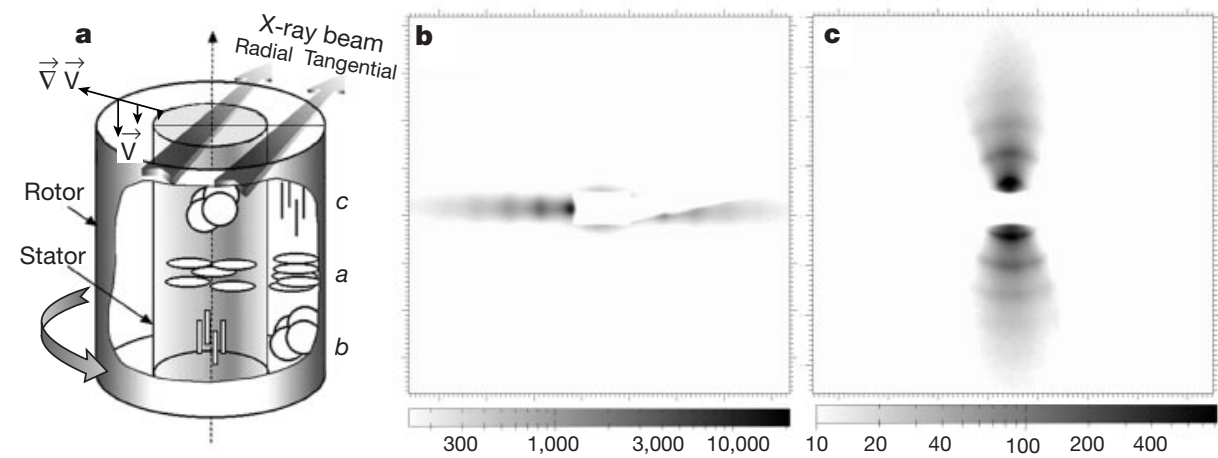

Figure 5 Small-angle X-ray scattering studies of aligned samples. a, Scheme of the Couette shear cell illustrating the radial and tangential geometries with respect to the $X$-ray beam (flat arrows). We also depict within the cell the three different types of lamellar orientations $(a, b$ and $c)$ as seen by the X-rays in both geometries (the layers are sketched as circular disks). b, SAXS two-dimensional scattering pattern obtained with a sample aligned in a Couette shear cell, in the tangential geometry, showing that the layers $(d=175 \mathrm{~nm})$ mostly belong to the $c$ orientation. c, SAXS two-dimensional scattering pattern of a vertical capillary filled with a suspension of $\mathrm{H}_{3} \mathrm{Sb}_{3} \mathrm{P}_{2} \mathrm{O}_{14}(\phi=0.75 \%)$, obtained 20 min after its alignment using a 18.7-T magnetic field applied along its long axis. The vertical localization of the interference peaks $(d=215 \mathrm{~nm})$ indicates that the layers are oriented perpendicular to the magnetic field (in this case, as no mechanical shear is applied, the sample relaxes back to a powder within 1-2 hours once it has been removed from the magnetic field). 
membranes formed by self-assembly ${ }^{26}$. The elastic constants and the diffraction peak profiles of single domains of this new phase should be carefully examined and compared to these predictions.

From the point of view of materials chemistry, the approach described here should lead to the discovery of a whole new class of mineral lamellar liquid crystalline phases. Indeed, from current work, we expect that similar phase behaviour will be found not only in other related members of the series $\mathrm{H}_{3} \mathrm{M}_{3} \mathrm{Z}_{2} \mathrm{O}_{14}$ (with for example $\mathrm{M}=\mathrm{Sb}, \mathrm{Nb}, \mathrm{Ta} ; \mathrm{Z}=\mathrm{P}, \mathrm{As})$, but also in phases such as $\mathrm{HM}_{2} \mathrm{Nb}_{3} \mathrm{O}_{10}$ $(\mathrm{M}=\mathrm{Pb}, \mathrm{Ca}), \mathrm{HTiNbO}_{5}$ or $\mathrm{Zr}\left(\mathrm{HPO}_{4}\right)_{2} \cdot \mathrm{H}_{2} \mathrm{O}$ that can all be fully exfoliated using tetra( $n$-butyl)ammonium hydroxide ${ }^{27,28}$. All of these phases, because of the robustness of their layers, could be used for polymerization of soluble species under tunable twodimensional confinement conditions.

Received 8 January; accepted 25 July 2001

1. Eigler, D. M. \& Schweizer, E. K. Positioning single atoms with a scanning tunneling microscope Nature 344, 524-526 (1990)

2. Poncharal, P., Wang, Z L., Ugarte, D. \& de Heer, W. A. Electrostatic deflections and electromechanical resonances of carbon nanotubes. Science 283, 1513-1516 (1999).

3. Blanco, A. et al. Large-scale synthesis of a silicon photonic crystal with a complete three-dimensional bandgap near 1.5 micrometres. Nature 405, 437-440 (2000).

4. Davidson, P., Gabriel, J.-C., Levelut, A.-M. \& Batail, P. Nematic liquid crystalline mineral polymers. Adv. Mater. 5, 665-668 (1993).

5. Gabriel, J.-C. P. \& Davidson, P. New trends in colloidal liquid crystals based on mineral moieties. $A d v$. Mater. 12, 9-20 (2000)

6. Kajiwara, K., Donkai, N., Hiragi, Y. \& Inagaki, H. Lyotropic mesophase of imogolite 1. Effect of polydispersity on phase diagram. Makromol. Chem. 187, 2883-2894 (1986).

7. Nikoobakht, B., Wang, Z. L. \& El-Sayed, M. A. Self-assembly of gold nanorods. J. Phys. Chem. B 104, 1346-1348 (2000).

8. Maeda, Y. \& Hachisu, S. Schiller layers in beta-ferric oxyhydroxide sol as an order-disorder phase separating system. Coll. Surf. 6, 1-16 (1983).

9. Brown, A. B. D., Ferrero, C., Narayanan, T. \& Rennie, A. R. Phase separation and structure in a concentrated colloidal dispersion of uniform plates. Eur. Phys. J. B 11, 481-489 (1999).

10. van der Kooij, F. M., Kassapidou, K. \& Lekkerkerker, H. N. W. Liquid crystal phase transitions in suspensions of polydisperse plate-like particles. Nature 406, 868-871 (2000).

11. Fraden, S. in Observation, Prediction, and Simulation of Phase Transitions in Complex Fluids (ed Baus, M., Rull, L. F. \& Ryckaert, J. P.) 113-164 (NATO ASI Series, Kluwer Academic, Dordrecht, 1995).

12. Whittingham, M. W. \& Jacobson, A. J. Intercalation Chemistry (Academic, New York, 1982).

13. Gabriel, J.-C. P., Sanchez, C. \& Davidson, P. Observation of nematic liquid-crystal textures in aqueous gels of smectite clays. J. Phys. Chem. 100, 11139-11143 (1996).

14. Mourchid, A., Lécolier, E., Van Damme, H. \& Levitz, P. On viscoelastic, birefringent, and swelling properties of laponite clay suspensions: revisited phase diagram. Langmuir 14, 4718-4723 (1998).

15. Piffard, Y., Verbaere, A., Lachgar, A., Deniard-Courant, S. \& Tournoux, M. The layered phosphatoantimonic acid $\mathrm{H}_{3} \mathrm{Sb}_{3} \mathrm{P}_{2} \mathrm{O}_{14}, \mathrm{x} \mathrm{H}_{2} \mathrm{O}$. Rev. Chim. Miner. 23, 766-775 (1986).

16. Boesecke, P., Diat, O. \& Rasmussen, B. High-brilliance beamline at the European Synchrotron Radiation Facility. Rev. Sci. Instrum. 66, 1636-1638 (1995).

17. Safinya, C. R. et al. Steric interaction in a model multimembrane system: a synchrotron study. Phys. Rev. Lett. 57, 2718-2721 (1986).

18. Salditt, T. et al. Specular and diffuse scattering of highly aligned phospholipid membranes. Phys. Rev. E 60, 7285-7289 (1999)

19. Deryagin, B. V. \& Landau, L. Theory of the stability of strongly charged lyophobic sols and of the adhesion of strongly charged particles in solutions of electrolytes. Acta Physicochim. URSS 14, 633 $662(1941)$

20. Verwey, E. J. W. \& Overbeek, J. Th. G. Theory of the Stability of Lyophobic Colloids (Elsevier Amsterdam, 1948).

21. Safinya, C. R. et al. Structure of membrane surfactant and liquid-crystalline smectic lamellar phase under flow. Science 261, 588-591 (1993).

22. Tjandra, N. \& Bax, A. Direct measurement of distances and angles in biomolecules by NMR in a dilute liquid crystalline medium. Science 278, 1111-1114 (1997)

23. Desvaux, H., Gabriel, J.-C. P., Berthault, P. \& Camerel, F. First use of a mineral liquid crystal for NMR measurement of residual dipolar couplings of a non-labeled biomolecule. Angew. Chem. 40, 373-376 (2001).

24. Ramirez, B. E. \& Bax, A. Modulation of the alignment tensor of macromolecules dissolved in a dilute liquid crystalline medium. J. Am. Chem. Soc. 120, 9106-9107 (1998).

25. Janiak, M. J., Small, D. M. \& Shipley, G. G. Nature of the thermal pretransition of synthetic phospholipids: Dimyristoyl- and Dipalmitoyllecithin. Biochemistry 15, 4575-4580 (1976).

26. Toner, J. New phase of matter in lamellar phases of tethered, crystalline membranes. Phys. Rev. Lett. 64, 1741-1744 (1990).

27. Fang, M. et al. Layer by layers growth and condensation reactions of niobate and titanoniobate thin films. Chem. Mater. 11, 1526-1532 (1999).

28. Kaschak, D. M. et al. Chemistry on the edge: a microscopic analysis of the intercalation, exfoliation, edge functionalization and monolayer surface tiling reactions of $\alpha$-zirconium phosphate. J. Am. Chem. Soc. 120, 10887-10894 (1998).

29. Kay, L. E., Keifer, P. \& Saarinen, T. Pure absorption gradient enhanced heteronuclear single quantum correlation spectroscopy with improved sensitivity. J. Am. Chem. Soc. 114, 10663-10665 (1992)
Supplementary information is available on Nature's World-Wide Web site (http://www.nature.com) or as paper copy from the London editorial office of Nature.

\section{Acknowledgements}

We thank the LURE and ESRF synchrotron radiation facilities for the award of beamtime, C. Bourgaux, P. Panine and T. Narayanan for technical support at D24 (LURE) and ID2 (ESRF), Y. Piffard for supplying us with $1 \mathrm{~g}$ of $\mathrm{H}_{3} \mathrm{Sb}_{3} \mathrm{P}_{2} \mathrm{O}_{14}$ powder at the start of this work, $\mathrm{C}$. Chaudemanche and $\mathrm{X}$. Leguevel for their participation in the synthesis of large amounts of $\mathrm{H}_{3} \mathrm{Sb}_{3} \mathrm{P}_{2} \mathrm{O}_{14}$, F. Alvarez for $\mathrm{pH}$ measurements, S. Grolleau for TGA measurements, P. Berthault and D. Jeannerat for their help in NMR measurements and processing of the spectra, P. Sinaÿ and Y. Zhang for the gift of the pentasaccharide and J. P. Simorre for giving us access to a 18.7 T magnet while we were performing our SAXS experiments at the ESRF. Financial support from the Ministry of Education (PhD fellowship for F.C.), the Ecole Normale Supérieure and the Ecole Nationale des Ponts et Chaussées (PhD fellowships for B.L.), the Région Pays de Loire and the GDR-CNRS 690 FORMES (for $\mathrm{D}_{2} \mathrm{O}$ ) is gratefully acknowledged.

Correspondence and requests for materials should be addressed to J.-C.P.G. (e-mail: jeang@covalentmaterials.com) or P.D. (e-mail: davidson@lps.u-psud.fr).

\section{High frequency of 'super-cyclones' along the Great Barrier Reef over the past 5,000 years}

\author{
Jonathan Nott* \& Matthew Hayne $\dagger$ \\ * School of Tropical Environment Studies and Geography, James Cook University, \\ PO Box 6811, Cairns, Queensland, 4870, Australia \\ $\dagger$ Australian Geological Survey Organisation, GPO Box 378, Canberra, \\ Australian Capital Territory, 2601 Australia
}

Understanding long-term variability in the occurrence of tropical cyclones that are of extreme intensity is important for determining their role in ecological disturbances ${ }^{1-5}$, for predicting present and future community vulnerability and economic loss ${ }^{6}$ and for assessing whether changes in the variability of such cyclones are induced by climate change ${ }^{7}$. Our ability to accurately make these assessments has been limited by the short (less than 100 years) instrumented record of cyclone intensity. Here we determine the intensity of prehistoric tropical cyclones over the past 5,000 years from ridges of detrital coral and shell deposited above highest tide and terraces that have been eroded into coarse-grained alluvial fan deposits. These features occur along $1,500 \mathrm{~km}$ of the Great Barrier Reef and also the Gulf of Carpentaria, Australia. We infer that the deposits were formed by storms with recurrence intervals of two to three centuries ${ }^{8-11}$, and we show that the cyclones responsible must have been of extreme intensity (central pressures less than $920 \mathrm{hPa}$ ). Our estimate of the frequency of such 'super-cyclones' is an order of magnitude higher than that previously estimated (which was once every several millennia $\left.{ }^{12-14}\right)$, and is sufficiently high to suggest that the character of rainforests and coral reef communities were probably shaped by these events.

In tropical, intraplate settings that are largely free from the effects of sizeable earthquakes, tsunami, volcanic eruptions and landslides, tropical cyclones rank as one of the main hazards affecting both natural and human communities. In natural communities, disturbance by hazards is one of the most important mechanisms affecting the evolution and diversity of species ${ }^{1-5}$. Because of the short timescales over which direct and continuous observation of change within ecological communities is possible ${ }^{5}$, it has been difficult to assess the influence of various scales of disturbance, and whether other mechanisms such as recruitment limitation ${ }^{15}$ are 